\title{
Power Aware Progressive Energy Routing Protocol for MANET
}

\author{
J.Nandhini \\ Research Scholar / ECE \\ Jay Shriram Group of Institutions, \\ India - 638660
}

\author{
D.Sharmila, Ph.D \\ Professor \& Head / EIE \\ Bannari Amman Institute of \\ Technology \\ India - 638401
}

\author{
K.K.Savitha \\ Associate Professor / MCA \\ KSR College of Engineering \\ India - 637215
}

\begin{abstract}
Mobile ad hoc networks are infrastructure-less networks used for communication between two or more nodes without a common access point. Due to frequent changes within the topology of the network, Routing protocol design with energy efficiency is an important issue. Our proposed scheme MPEER integrates Energy efficient routing scheme to improve the performance during path discovery and in mobility scenarios. A discrete level of power control and priority based packet scheduling is also done. The proposed routing method achieves energy efficient routing by considering shortest path, avoids retransmission with reduced overhead and path setup delay as compared to other conventional routing protocols.
\end{abstract}

\section{Keywords}

Adhoc network, Energy efficiency, MPEER protocol, Capacity maximization

\section{INTRODUCTION}

Mobile Adhoc network is a self-configuring network of wireless links connecting mobile nodes. Each node is free to move on its own and all nodes organize themselves in an arbitrary fashion. There is no static infrastructure needed such as base station. Each node must work as a router and forward messages to the appropriate device. When the Source and Destination nodes are not within their range, then the message or packet communication between them must be done only by relying on the intermediate nodes. MANETs support some specific applications like virtual lecture rooms, military communications, emergency search and rescue operations, information acquisitions in hostile environments, communication setup in exhibitions, conferences, in battlefields among troopers to coordinate defense or attack, at field terminals for staff to share files etc [1].

Nodes in an Adhoc network are constrained by limited battery power for their operation. Energy management is an important issue in such networks. Battery power is a precious resource that must be used efficiently in order to avoid early termination of nodes which lead to path breakup. Energy saving issues start by saving power by reducing the control messages which are used to assert neighbor relationships and synchronization purposes [2]. Designing good protocols with few packet collisions reduces power consumption since retransmission of packets requires energy. Network lifetime is increased by attempting to reduce node and / or path failure. Hence the lifetime of nodes and the network has become an important objective.

In this work, Minimum energy routing protocol is considered to improve the performance during path discovery and in mobility conditions. This is combined with new on demand power aware route search scheme to provide the efficient route within given route search delay bound. By using minimally required level of transmission power for each hop, proposed route search scheme can increase battery life of the nodes, and also increase network capacity by reducing interference range.

In section 2 presents the routing protocol classification, section 3 gives the related works, section 4 addresses the proposed method MPEER with capacity maximization techniques, section 5 gives the simulation results and section 6 concludes the paper.

\section{RELATED WORK}

To conserve energy, many energy-efficient routing protocols have been proposed. Here we will discuss some of the protocols.

Flow Augmentation Routing (FAR), Online Max-Min Routing (OMM), Power Aware Localized Routing (PLR) and Minimum Energy Routing (MER). These Protocols were used to minimize the total transmission energy but avoid low energy nodes [4]. Protocols such as Re-transmission- energy aware routing (RAR) and Smallest Common Power routing (COMPOW) [5] considered the reduction in Total transmission energy by considering retransmission overhead or bi-directionality requirement.

In case of Load Distribution approach, Localized Energy Aware Routing (LEAR) and Conditional Max-Min Battery Capacity Routing (CMMBCR) [6] were used to distribute load to energy rich nodes. 
Sleep / Power down mode uses SPAN, Geographic Adaptive Fidelity (GAF), Prototype Embedded Network (PEN) to minimize energy consumption during inactivity [7].

Jihui Zhang et al propose energy efficient routing in a multihop wireless network by considering mobility into account using a deterministic model. They minimize the energy consumption for packet delivery with reduced packet delay for concurrent transmissions with heuristic approach [8].

Xiang-Yang $\mathrm{Li}$ et al suggests protocol for routing in the presence of unreliable communication links or devices by integrating the power control techniques into the energy efficient routing in unicast and multicast scenarios [9]. In this method, when there is only one common source node, power assignment and routing are optimal. For multicast routing protocol, energy consumption is no more than two times of the minimum in a one-to-one communication model.

Humaira Nishat et al propose Minimum Maximum Battery cost Routing Protocol in which the route selection is based on the battery capacity of all the individual nodes [2]. This protocol first finds the node with minimum battery capacity in each of the possible routes and having maximum value among the selected routes i.e., the route with maximum lifetime is selected.

Sunho Lim et al propose Randomcast mechanism to avoid unnecessary energy wastage of nodes energy through overhearing. In this method, when a packet is transmitted, nodes in the proximity should decide whether or not to overhear it considering the tradeoffs between energy efficiency and routing efficiency [10]. This method also avoids redundant rebroadcasts and thus saves additional energy.

Bulent Tavli et al propose MC-TRACE, a cross layer energyefficient real time data multicasting architecture [11] for Adhoc networks. MS-TRACE is targeted at reducing the total energy dissipation, which consists of not only transmit energy dissipation, but also receive, carrier sense, idle and sleep energy dissipations as well. Delay is higher and Jitter is lesser compared to ODMRP.

\section{PROPOSED WORK}

The limited resources in MANET have made designing of an efficient routing a challenging problem. An intelligent routing strategy is needed to efficiently use the restricted resources whereas at constant filmable to the dynamical network conditions such as: size of the network, traffic density and partitioning of the network. The routing protocol might have to produce completely different levels of QoS to differing kinds of applications and users. At any point of time the wireless network node will be in any one of the following four states: Transmit Receive, Idle or Sleep. For each state a different level of energy consumption will be there :
Transmit: To transmit data or control packets each node needs some transmission power.

Receive: To receive data or control packets each node needs some reception power. Even if the packet received will be discarded by the node when the packets are not decoded correctly or forwarded since it is not intended for current node.

Idle (listening): The nodes stay at rest even when no messages are being transmitted over the medium and keep listening the medium.

Sleep: When the node is not capable of detecting signals, no communication is possible its turn off to save energy.

This paper presents the integration of Progressive Energy Efficient Routing Protocol (MPEER) and capacity maximization technique with scheduling mechanisms. The MPEER approach establishes energy efficient path during route discovery process and maintains the route actively so as to respond to topology and channel changes quickly [12]. To reduce the quantity of excess rebroadcasts, and guarantee that the packet containing the foremost energy economical route arrives at the destination each node controls the time of rebroadcast by using a priority based packet scheduling [13].

\subsection{Routing Mechanism using MPEER}

The proposed scheme considers on demand routing along with the threshold value of nodes. The quickest way to find a path between two nodes would be through a shortest path routing scheme. There may exist a few shortest paths between source node and destination node. Among the accessible shortest paths, it's higher to choose the foremost energy - efficient one. The algorithm starts by searching for all shortest paths and pick the minimum energy conservation path among the shortest path

Route discovery process is initiated for packet transmission from source node to destination node. In this process, the source node sends a RREQ message to destination node through the shortest path. The paths were found out by considering the link cost (hop count) and energy (battery power) of the neighboring nodes. The link cost and energy is compared with the threshold value (energy) of the source node.

When the energy of the source node is greater than the combined link cost and energy of the neighboring nodes, node will not be considered for routing and RREQ messages may be forwarded to other neighboring nodes. On comparing again with the same conditions, and if the source node energy is found to be lesser than the energy and link cost of the neighboring nodes, the nodes will be consider as one of the node for shortest path. The same procedure is repeated for finding the shortest path among all nodes in the network. When the path is established, Route Reply (RREP) messages are forwarded though the same reverse route and the path is established for transmission. 


\subsection{Priority based packet Scheduling}

Once the path is established by means of Modified PEER routing process, transmission power level of intermediate nodes are measured and stored into the route request packet with its own ID and rebroadcast the route request packet. To reduce the quantity of surplus rebroadcasts, and guarantee that the packet containing the foremost energy economical route arrives at the destination, each node controls the time of rebroadcast by using priority based packet scheduling. This process uses link-based information to represent dynamic network environment.

At an intermediate node, determined transmission power level $\mathrm{L}_{\mathrm{i}}\left(\mathrm{p}_{\mathrm{t}}\right)$ is used as a basis of packet scheduling for rebroadcast. Scheduling time is calculated by

$\mathrm{T}_{\text {scheduled }}=\mathrm{T}_{\text {now }}+\mathrm{k}\left(\mathrm{L}_{\mathrm{i}}\left(\mathrm{P}_{\mathrm{t}}\right)\right)^{\mathrm{n}}$

where $\mathrm{k}$ is a scaling factor and $\mathrm{n}$ is the path loss coefficient. The scheduling process works as follows. If an arrived route request packet has the same packet id previously broadcasted, then the scheduler drops the packet. If the calculated time is later stored one in the delay buffer, the scheduler drops the packet. Only if the latterly arrived packet has sooner scheduled time than any other packet which has same packet id, the packet goes into the delay buffer. As time goes by, a route request packet having the shortest scheduled time is selected and passed to routing module by the scheduler. As a result, most energy efficient route is always selected in the various node distribution patterns.

\subsection{Proposed Algorithm}

The proposed algorithm aims to extend the network lifetime and minimize the energy consumption throughout the source to destination route establishment. The proposed work provides energy economical path between a source and destination pair. The designed algorithm has been implemented on AODV.

\section{SOURCE NODE:}

Check in routing table for destination location

IF it is not available THEN

Initiate route discovery process

Enclose Destination ID and its energy

Enclose minimum power required to transfer the packet // Threshold

And then broadcast packet

Store data in buffer

ELSE IF path available for destination Forward packet

Choose next hop based on required power

\section{ENDIF}

IF Source receives RREP THEN

Send stored packet to path

Delivery the packet from buffer

Data Transmission

\section{ENDIF}

\section{INTERMEDIATE NODE :}

Verify Signal Strength for coverage Based on RSS classify node power if $($ rss_category $==1$ )
\{

node_power_list_.add(ch->prev_hop_,HI_PWR);

\}

Else if( rss_category == 2 )

node_power_list_.add(ch->prev_hop_,AVG_PWR_1);

Else if(rss_category $==3$ )

node_power_list_.add(ch->prev_hop_,AVG_PWR_2);

Else

\{

node_power_list_.add(ch->prev_hop_,LOW_PWR);

Verify its energy level

IF min transmission power available THEN

compute schedule time

check sequence number and route request id for freshness

check the timer for reply message

send route reply message with available paths

ENDIF

DESTINATION NODE :

// When RREQ came to the Destination node

IF Dest_ID = Node_ID THEN

Energy level of current node is >= the AVG_PWR

RREP is sent to the selected path.

ENDIF

Figure 1. Algorithm for Energy aware Route Discovery

\section{SIMULATION}

\subsection{Simulation Environment}

The simulation setup consists of square flat topology covering an area of $500 * 500 \mathrm{~m} 2$ and a test bed of 50 nodes randomly and uniformly distributed in the entire area. The number of wireless mobile nodes is fixed to 50. The random waypoint model is used to model mobility. All random scenarios have been generated for a maximum speed of $4 \mathrm{mtr}$ /s with a pause time of 10 seconds. Traffic sources are chosen as CBR with a packet size of 512 bytes. All traffic sessions are established at random times near the beginning of the simulation run and they remain active until the end of the simulation period. A constant transmission range of $250 \mathrm{~m}$ is considered for all RREQ packets. The transmission range and power varies with distance between two nodes when actual data transfer occurs after route establishment. Each of the 20 nodes has 100 Joules of energy at the start of every simulation. The number for traffic connections was taken as 6 . Identical mobility and traffic scenarios are used across the protocol variations. Track of residual energy of a node is done by using in an inbuilt energy model of NS - 2 that uses a traditional method of keeping track of the residual energy of a node. 


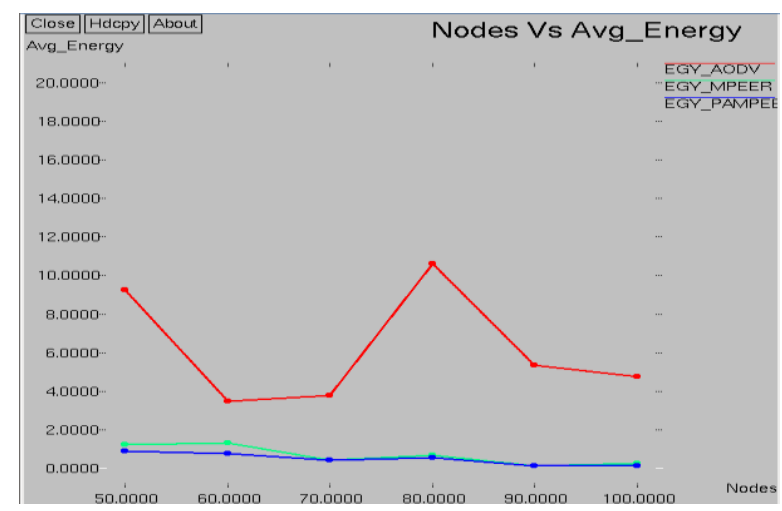

Figure 2. Average Energy Consumption

Figure 2 show the simulation results achieved for Power aware Modified PEER as compare with AODV and MPEER. When the number of nodes is varied, the proposed method consumes less energy than the remaining routing process.

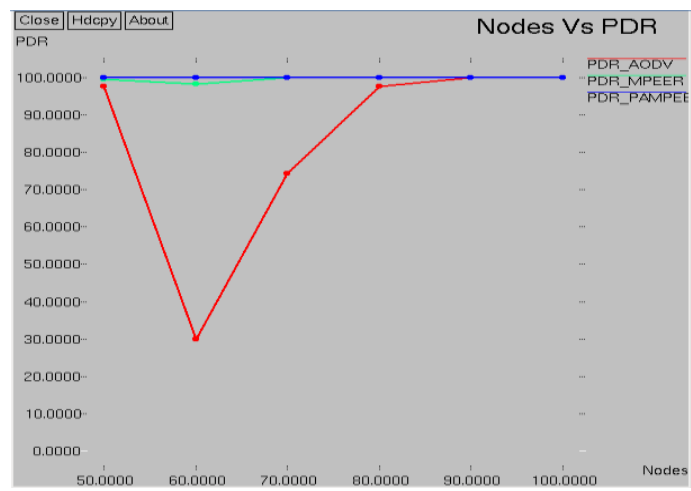

Figure 3. Packet Delivery Ratio

Figure 3 presents the packet delivery ratio achieved by varying the number of nodes in the simulation environment. Results show that, the proposed method achieves better packet deliver ratio than the remaining routing process.

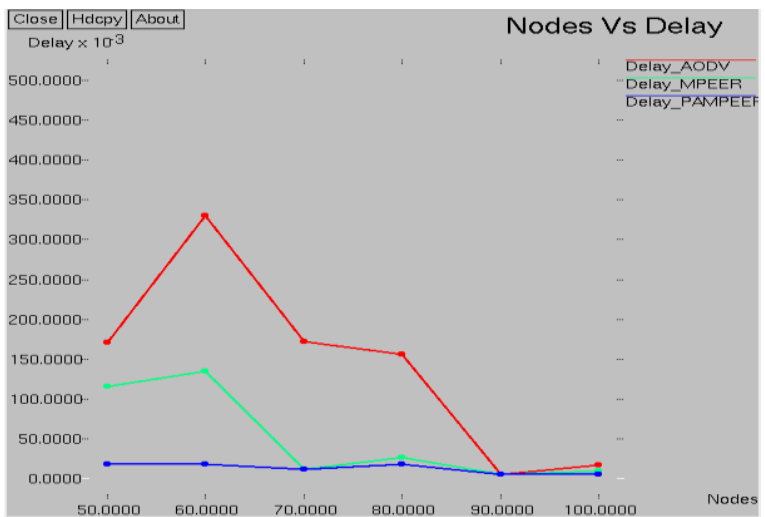

Figure 4. Average Delay

Figure 4 show the simulation results achieved for Power aware Modified PEER as compare with AODV and MPEER. Results show that, the proposed method has reduced delay than the other routing process.
Network throughput is the amount of data successfully sent and received (without errors) by the entire network within the simulated data transfer time. In energy terms, the higher the throughput the better performing the protocol

Nodes ensuring high network throughput are considered optimum with their energy sources, meaning that the underlying protocol algorithm is running efficiently.

Low throughput is the amount of data successfully sent and received (without errors) by the entire network within the simulated data transfer time. In energy terms, higher throughput implies better performance of the protocol.

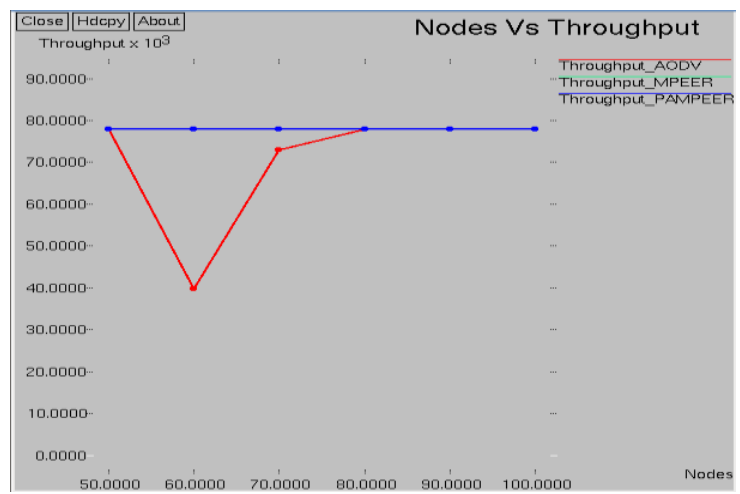

Figure 5. Average Throughput

Figure 5 show the simulation results achieved for Power aware Modified PEER as compare with AODV and MPEER. Since the maximum number of packets reaches the destination, the proposed method achieves higher throughput than the other method.

\section{CONCLUSION}

This paper proposes Modified Progressive Energy Efficient Routing (MPEER) with Scheduling mechanisms for energy efficient operation. The proposed scheme integrated the advantages of Modified PEER by choosing shortest and energy efficient path using link cost and avoids rebroadcast by priority based scheduling process. From the simulated results it is observed that the proposed method works well by consuming less energy for path setup and delay is minimized. The average number of packets transmission and reception is also better compared to AODV.

Energy efficiency is one of the main problems in a mobile ad hoc network, especially designing a routing protocol. The proposed work aims at discovering an efficient energy aware routing scheme in MANETs that not only uses the node energy effectively but also finds the best route and increases the lifetime of the network. Simulation result shows that the proposed scheme outperforms in terms of different energy related parameters over AODV even in high mobility scenarios. At the time of route selection, the proposed algorithm takes care of crucial things, battery status of the path, and number of drained nodes in the path. 
With these factors in consideration the proposed scheme always select more stable route for data delivery. Energy of the network is also reduced using variable transmission power when data transmission is done. The results obtained from implementing these techniques are favorable and encouraging. Performance evaluation using a NS-2.34 shows that the longevity of the network can be extended by a significant amount. For the chosen simulation parameter set, in low traffic scenario, the average residual energy of the network is increased by $30-40 \%$. For high traffic scenarios this goes up by $45 \%$. Thus results obtained indicate that proposed scheme outperforms AODV for low and heavy traffic scenarios. This is mainly due to the fact that the proposed scheme analyses various route energy before selecting a route for transmission. On the contrary, AODV selects the quickest route irrespective of the route energy. Hence, its network lifetime is reduced significantly when compared with proposed scheme. The proposed energy-aware scheme can be further studied and analyzed for different parameters in scarce medium.

\section{REFERENCES}

[1] Madhukar Rao, M.Nadeem Baig, Md.Fareed Baba, K.Kanthi kumar, 2011.Energy Efficient Reliable Routing Protocol for Mobile Ad hoc Networks, IEEE.

[2] Humaira Nishat, Dr.D.Srinivasa Rao, Dr.Ch.Balaswamy, 2011. Energy Efficient Routing Protocols for Mobile Adhoc Networks, International Journal of Computer Applications, Volume 26- No.2.

[3] Victor Govindaswamy, William L.Blackstone, G.Balasekaran, 2011. Survey of Recent Position Based Routing Mobile Adhoc Network Protocols, International conference on Modeling and Simulation.

[4] Chang J-H, Tassiulas L, 2000. Energy Conserving Routing in Wireless Ad-hoc Networks. Proceedings of the Conf. on Computer Communications, IEEE; 2000.

[5] Banerjee S, Misra A, 2002. Minimum Energy Paths for Reliable Communication in Multi-hop Wireless Networks, Proceedings of Annual Workshop on Mobile Ad Hoc Networking \& Computing.
[6] Toh C-K, 2001. Maximum Battery Life Routing to Support Ubiquitous Mobile Computing in Wireless Ad Hoc Networks, IEEE Communications.

[7] Girling G, Osborn P, Stefanova R, 2000. The Design and Implementation of a Low Power Ad Hoc Protocol Stack, Proceedings of IEEE Wireless Communications and Networking Conference.

[8] Jihui Zhang, Zhang, Bo Li, Xiaonan Luo, Wenwu Zhu, 2006. Energy-Efficient Routing in Mobile Ad Hoc Networks: Mobility-Assisted Case, IEEE Transactions On Vehicular Technology.

[9] Xiang-Yang Li, Yu Wang, Haiming Chen, Xiaowen Chu, Yanwei Wu, Yong Qi, 2009. Reliable and EnergyEfficient Routing for Static Wireless Adhoc Networks with Unreliable Links, IEEE Transactions On Parallel And Distributed Systems, Vol. 20, No. 10.

[10] Sunho Lim, Chansu Yu, Chita R. Das, 2009. RandomCast: An Energy-Efficient Communication Scheme for Mobile Ad Hoc Networks, IEEE transactions on mobile computing, Volume 8, No 8.

[11] Bulent Tavli, Wendi B.Heinzelman, 2011. EnergyEfficient Real - Time Multicast Routing in Mobile Adhoc Networks, IEEE Transactions on Computers, Vol 60, No 5 .

[12] Jinhua Zhu, Xin Wang, 2011. Model and Protocol for Energy Efficient Routing over Mobile Adhoc Networks, IEEE transactions on mobile computing, Volume 10, No 11.

[13] Dr.R.K.Chauhan, Ashish Chopra, 2010. Energy Efficient Routing in Mobile Adhoc Network with Capacity Maximization, IJCA Special Issue on Mobile Adhoc Networks. 